\section{Chromatin's rising tide}

\section{By Chris Cain, Senior Writer}

To capitalize on the full range of possible chromatin targets in and beyond oncology, industry and academia will need to delve deeper into how chromatin regulation is altered in disease, create tools that can reliably validate new targets and develop biomarkers that can improve the chances of success in clinical trials.

A wave of compounds targeting chromatin regulators entered the clinic in 2013, enabled by a decade of progress in understanding how chromatin dysfunction drives cancer. ${ }^{1}$

The first DNA methyltransferase (DNMT) and histone deacetylase (HDAC) inhibitors were approved to treat subtypes of lymphoma in 2004 and 2006, respectively. In the following decade, research linked genetic alterations in functionally diverse chromatin regulators to many additional types of cancer.

That fueled the discovery of a second generation of compounds against new targets to treat genetically defined cancers (see Table 1, "Select clinical-stage compounds that target epigenetic regulators").

Development of this wave of compounds was aided by technical advances that allow chromatin to be biochemically characterized in detail. Despite the progress, there remains a huge opportunity in unexplored targets and much to discover about how chromatindependent cellular pathways are affected in different diseases.

Against this backdrop, SciBX organized a panel of thought leaders to discuss the possibilities and challenges in developing chromatintargeted compounds and outline ways to accelerate the translation of this information into disease-modifying therapies.

The panel identified three areas of chromatin drug development that are most in need of innovation.

First, new chemical tools to functionally characterize chromatin will be critical to validate targets-including those currently deemed intractable. These tools will enable a deeper understanding of how mutations in chromatin regulators alter cell signaling pathways and cell fate.

Panel member Jim Audia, CSO at Constellation Pharmaceuticals Inc., said that the optimal way to generate new tool compounds would be through collaborations between industry and academia such as the Structural Genomics Consortium (SGC).

"We realize that, regardless of your company, the academic community from outside it is immense compared to the resources that you can muster from within," he said.

Second, identifying predictive biomarkers and developing new methods to measure target engagement in vivo will be needed to accelerate progress to the clinic. Information about how hitting chromatin targets provokes different cellular responses between individuals will help companies select appropriate patient populations.

Because little is known about how the cellular pathways involved in chromatin regulation differ cell by cell or tissue by tissue, that information will need to be integrated from multiple experimental approaches.

"The simplest readout, if you were to perform knockdowns or if you had an inhibitor, would just be to look at changes in gene expression," said Peter Tummino, who at the time of the panel was head of GlaxoSmithKline plc's Cancer Epigenetics Discovery Performance Unit (DPU). "But what we are finding is that it is entirely insufficient. You can look across a set of cell lines and there's no similarity, there is no common gene profile. And so the next step beyond that is to think about what are the changes in histone marks-which you might expect to change. You can perform transcription factor mapping, if possible. It's the integration of several different data sets that begins to lead toward an understanding of mechanism.”

Tummino is now VP and global head of lead discovery at the Janssen unit of Johnson \& Johnson.

Finally, the panel said that chromatin regulators should also be targeted outside of oncology. Human genetic studies and an increased understanding of how cell fate is determined in neurology and immunology suggest that these two fields are next in line.

"We're years behind the oncology field in terms of looking at target association in the CNS," said Ankit Mahadevia, venture partner at Atlas Venture and acting CBO of Rodin Therapeutics Inc. "We're not wanting for genetically implicated targets in terms of CNS applications. Really, it's a lack of chemical probes and high-fidelity assays. The field is just so nascent, and there are fewer people, both in academia and in pharma, working on these associations."

Audia, Tummino and Mahadevia participated in the panel discussion alongside Jesse Smith, executive director of biological sciences at Epizyme Inc.; Charles Roberts, an associate professor of pediatrics at Harvard Medical School and director of the research program in solid tumors at the Dana-Farber Cancer Institute; and Stuart Schreiber, director of the Center for the Science of Therapeutics at the Broad Institute of MIT and Harvard, director of chemical biology at the Broad Institute, a professor of chemistry and chemical biology at Harvard University and an investigator at the Howard Hughes Medical Institute.

The panel was part of the 2nd SciBX Summit on Innovation in Drug Discovery \& Development and was produced with support from the following sponsors: Acetylon Pharmaceuticals Inc., Amgen Inc., AstraZeneca plc, Atlas Venture, Biogen Idec Inc., GlaxoSmithKline, Karus Therapeutics Ltd., Merck \& Co. Inc., Novartis AG, RaNa Therapeutics Inc., Sanofi and Zenith Epigenetics Corp.

\section{Rational advancement}

The first DNMT and HDAC inhibitors were developed when knowledge of chromatin regulation was sparse at best. Indeed, compounds that inhibit these targets were shown to have anticancer properties. Only later were their mechanisms of action established. 


\begin{tabular}{|c|c|c|c|c|}
\hline Company & Compound & Description & Indication & Status \\
\hline Resverlogix Corp. (TSX:RVX) & RVX-208 & BET bromodomain inhibitor & Atherosclerosis & Phase II \\
\hline Acetylon Pharmaceuticals Inc.; Celgene & $\begin{array}{l}\text { Rocilinostat } \\
\text { (ACY-1215) }\end{array}$ & $\begin{array}{l}\text { Oral selective histone deacetylase } 6 \\
\text { (HDAC6) inhibitor }\end{array}$ & Multiple myeloma (MM) & Phase I/II \\
\hline $\begin{array}{l}\text { Epizyme Inc. (NASDAQ:EPZM); Eisai Co. Ltd. } \\
\text { (Tokyo:4523) }\end{array}$ & EPZ-6438 & $\begin{array}{l}\text { Enhancer of zeste homolog } 2 \\
\text { (EZH2) inhibitor }\end{array}$ & $\begin{array}{l}\text { Lymphomas including those with } E Z H 2 \\
\text { mutations }\end{array}$ & Phase I/II \\
\hline $\begin{array}{l}\text { Oryzon Genomics S.A.; Roche (SIX:ROG; } \\
\text { OTCQX:RHHBY) }\end{array}$ & ORY-1001 & $\begin{array}{l}\text { Lysine-specific demethylase } 1 \\
\text { (KDM1A; LSD1) inhibitor }\end{array}$ & Acute myelogenous leukemia (AML) & Phase I/IIa \\
\hline Constellation Pharmaceuticals Inc. & CPI-0610 & BET bromodomain inhibitor & Lymphoma & Phase I \\
\hline Epizyme; Celgene & EPZ-5676 & $\begin{array}{l}\text { Histone methyltransferase DOT1L } \\
\text { (DOT1L) inhibitor }\end{array}$ & $\begin{array}{l}\text { Myeloid-lymphoid or mixed-lineage } \\
\text { leukemia (MLL; HRX)-rearranged } \\
\text { leukemia }\end{array}$ & Phase I \\
\hline \multirow{3}{*}{ GlaxoSmithKline } & GSK2879552 & LSD1 inhibitor & Small cell lung cancer & Phase I \\
\hline & GSK525762 & BET bromodomain inhibitor & $\begin{array}{l}\text { Cancers including NUT (nuclear protein } \\
\text { in testis; C15orf55) midline carcinomas }\end{array}$ & Phase I \\
\hline & GSK2816126 & EZH2 inhibitor & $\begin{array}{l}\text { Lymphomas including those with } \mathrm{EZH} 2 \\
\text { mutations }\end{array}$ & Phase I \\
\hline $\begin{array}{l}\text { Oncoethix S.A.; Mitsubishi Tanabe Pharma Corp. } \\
\text { (Tokyo:4508) }\end{array}$ & OTX015 & BET bromodomain inhibitor & Hematologic malignancies & Phase I \\
\hline Tensha Therapeutics Inc. & TEN-010 & BET bromodomain inhibitor & $\begin{array}{l}\text { Cancers including NUT midline } \\
\text { carcinomas }\end{array}$ & Phase I \\
\hline
\end{tabular}

Since then, advances in whole-genome analysis and in vitro enzymology have ramped up knowledge about new enzymes and regulatory pathways and laid the groundwork for rational drug design.

According to the SGC, the key to exploiting this knowledge is a concerted chemical biology effort to probe the function of chromatin regulatory complexes in disease states. ${ }^{2}$

An example of the shift from phenotypic screening to rational design is the progress made since Schreiber's work in the mid-1990s that identified the mechanism of action of trapoxin, a natural product with anticancer activity that alters cell morphology.

Schreiber's 1996 study published in Science showed that the compound inhibited a previously uncharacterized protein that became known as HDAC $1 .^{3}$ At about the same time, independent teams identified the first mammalian histone acetyltransferase (HAT).

Now there are at least 18 known HDACs in humans, and companies including Acetylon and Karus are designing selective HDAC inhibitors.

Hundreds of additional proteins modify or alter chromatin structure. According to the SGC, there are 64 protein methyltransferases, two distinct families of protein demethylases and a slew of structurally distinct protein domains that bind acetylated or methylated histones or can physically remodel chromatin structure.

Schreiber said that the activities of chromatin-regulating protein families can be viewed as a natural extension of the signal transduction cascades that have been seen as potential drug targets for years.

"In signal transduction there are kinases that put on a phosphate mark, there are $\mathrm{SH} 2$ domains that bind the mark and there are phosphatases that remove the mark. And in chromatin it's exactly the same story. I'm a little amused that we have obfuscated this connection by using new vocabulary to describe proteins that behave like kinases and phosphatases. We call kinases 'writers', phosphatases are now 'erasers' and SH2 domains are 'readers.'
Schreiber said that compounds that target chromatin-modifying proteins have commonly been referred to as epigenetic drugs despite the fact that there is little evidence they would meet the traditional definition of epigenetics, in which an effect is inherited and persists across multiple generations. "This is not just semantics; it is medically relevant," he said.

For transient changes in chromatin, Schreiber added, there are clinical applications in which the effects would be seen rapidly. On the other hand, heritable changes in chromatin can involve cell fate and cellular differentiation, and drugs for those chromatin regulators could be effective in diseases of cellular deficiency.

Roberts said that genome sequencing has been central to linking chromatin-regulating enzymes to disease biology.

"I think the field really began to change when genome sequencing studies across cancers came out," said Roberts. "I think few people would have predicted that mutations in chromatin regulators and modifiersreaders, writers and erasers - would be prevalent in cancer before those studies were done, but that's precisely what was found."

Roberts noted that one complex in particular has turned out to be frequently mutated in cancers-members of SWI/SNF (switch/sucrose nonfermentable) chromatin remodeling complexes.

"Eight different subunits of this complex are recurrently mutated in cancer. The latest data suggest that $20 \%$ of all human cancers have a mutation of one or another SWI/SNF subunit. Furthermore, the genes encoding these subunits are being validated as bona fide tumor suppressors using mouse models. Now, an important question is what can we do about it therapeutically?" asked Roberts.

SWI/SNF is also an example of how hard it can be to convert genetic findings to new drug candidates when so little is known about their precise mechanism. 
Multiple independent groups including Roberts' have shown that components of the SWI/SNF complex can be targeted in cancers harboring mutations in BRG1 (SWI/SNF-related matrix-associated actin-dependent regulator of chromatin subfamily a member 4; SMARCA4) or ARID1A (AT rich interactive domain 1A), two of the complex's subunits.

But-as Smith pointed out-determining the best approach for targeting SWI/SNF complex members as drug targets is a difficult question because they can have multiple functions within the cell, including depositing chromatin marks, binding histones and facilitating proteinprotein scaffolding interactions.

In addition, the SWI/SNF complexes are large, multiprotein complexes with a diverse set of configurations based upon the various proteins that can comprise these assemblies. This phenomenon poses a significant challenge to biochemical and biophysical assay development for these targets.

For example, knockdown of BRM (SMARCA2) kills BRG1-mutant cancer cells, but it is not yet known whether small molecules that specifically target the protein's bromodomain region will reproduce the effect. ${ }^{4}$

\section{Chemicals matter}

There was wide agreement among panel members that the field needs more and better chemical probes to understand how dysfunctional chromatin regulators contribute to disease.

"I think the power of the probes has been demonstrated on many occasions, and I think there's clearly room for additional probes," said Audia. "If you look at the SGC, or you look across the industry, we're getting a fair number of probes targeting bromodomains and a few targeting select SET domain-containing proteins. We're starting to get a few examples for the demethylases. But there are vast classes of these chromatin regulators and modifiers that do not have adequate probes for even doing rudimentary experiments."

SET is the catalytic domain found in many lysine methyltransferases.

Thus far, the SGC has made available 18 probes targeting chromatin regulators. One of the most recent, the chemical probe PFI-3, inhibits the bromodomains of BRM, BRG1 and polybromo 1 (PBRM1; PB1). Although the chemical properties of the compound are available on SGC's website, no studies detailing functional data for PFI-3 have been published.

The ability of a probe to shape a therapeutic space was best demonstrated by the rapid pace of development of BET bromodomain inhibitors since the first probe was made available in 2011. BET bromodomain inhibitors have been critical for target validation in NUT (nuclear protein in testis; C15orf55) midline carcinoma and additional cancers. ${ }^{5,6}$

At least five BET bromodomain inhibitors are now in the clinic (see Table 1). These include TEN-010, a compound being developed by Tensha Therapeutics Inc. that was synthesized by James Bradner's lab.

His lab also synthesized the widely used research tool BET inhibitor JQ1 in collaboration with the SGC. ${ }^{5}$

Bradner is an attending physician in the Department of Medical Oncology at the Dana-Farber Cancer Institute, an assistant professor in the Department of Medicine at Harvard Medical School and associate director of the Center for the Science of Therapeutics at the Broad Institute.

All panelists agreed that more probes are needed but noted that the field needs to focus on generating high-quality probes that work across multiple assays and both in vitro and in vivo.

"If you get poor probes, you generate an awful lot of data that tends to confound the conclusions that are reached. I think that's one of the things that we spend a great deal of time trying to do-to thoroughly characterize all of the probes that we utilize internally in order to determine whether they selectively inhibit the appropriate target in a cellular context," said Smith.

He added, "There are plenty of examples of published inhibitors that have good biochemical potency and selectivity, but for various reasons they lack specific cellular target engagement, at least as measured by global or local methyl mark changes. If you treat cells with high enough concentrations of these inhibitors, you often will observe phenotypic effects, which are most likely due to off-target activity. So I think it is important for investigators in our field to be very clear about how their probes should and should not be used experimentally: can they be used in cell-based assays? Can they be used in animal studies? What are appropriate concentrations to use?"

Multiple panelists said that compounds with defined in vitro effects may behave differently inside cells. "We need compounds to do what we think they're doing, and we need to be certain of it," said Schreiber. "If we look to the kinase world, we've learned that, over and over, compounds work on their targets in cells in ways that can be context dependent. That's not seen biochemically in vitro."

Schreiber told SciBX that investigating new target classes may require creating new chemical compounds to screen against. "Screening collections are limited in what they can do, so a lot of this starts from getting the right chemistry," he said.

Schreiber is a cofounder of Forma Therapeutics Holdings LLC, which is developing diversity-oriented synthesis technology that the Broad Institute uses.

Mahadevia said that new chemical matter will be especially important in indications beyond oncology in which toxicity is less tolerable and the bar is higher for achieving an acceptable therapeutic index.

For example, he said, "one of our programs at Rodin is an isoformspecific HDAC inhibitor being developed to enhance cognition. It's a case where the biology has been under discussion for about half a decade but no one has been able to identify that exquisite isoform specificity that correlates with the better safety profile."

Karus CEO Simon Kerry added that in the case of HDAC6, it is clear that the enzyme acts on different substrates inside and outside of the nucleus, which highlights how it can be challenging to understand the cellular biology underlying the therapeutic effect of HDAC6 inhibitors even when they are highly selective.

Karus is developing selective HDAC6 inhibitors to treat cancer and inflammation, and Acetylon is developing selective HDAC inhibitors in both oncology and non-oncology indications. 


\section{Signature effects}

Another major challenge highlighted by the panelists is the lack of predictive biomarkers that could help explain the cellular effects of inhibiting particular chromatin targets. That lack of biomarkers has made it difficult to expand into cancers with no known genomic alterations.

Schreiber noted BET inhibitors as an example. "I still have not seen any predictor of response to BET inhibition other than the hematopoietic lineage or if BET itself is genomically altered. That's it, basically. The exception might be this very intriguing notion of these super-enhancers," he said.

Super-enhancers are a class of regulatory elements that control the expression of genesincluding oncogenes-involved in determining

"If you understand the function of a chromatin protein, there's a path forward."

- Peter Tummino, GlaxoSmithKline plc example of a program against a chromatin-modifying target that was not guided by the discovery of genomic alterations but instead by studies of the function of the protein in normal cells.

"In really nice work, LSD1 was shown to have a direct role in pluripotency and differentiation in hematopoietic cells. It was not a big leap then to at least hypothesize you'd have a similar role in acute myelogenous leukemia (AML). And it gives you greater confidence if you understand the role of the protein in its normal context," he said. "First Oryzon showed it with Tim Somervaille, and we've seen the same with our LSD1 inhibitor."

"If you're affecting the biology of a tumor type, and if you understand the function of a chromatin protein, there's a path forward," he said.

GSK's LSD1 inhibitor, GSK2879552, is in Phase I trials to treat small cell lung cancer. Oryzon Genomics S.A.'s LSD1 inhibitor, ORY-1001, is in Phase I/IIa testing to treat AML. In 2012, Somervaille, group leader of the Leukaemia Biology Laboratory at the Cancer Research UK Manchester Institute, published work in Cancer Cell using an LSD1 inhibitor designed by Oryzon. ${ }^{14}$

In April, Oryzon granted Roche exclusive worldwide rights to its LSD1 inhibitor program.

Tummino also noted that understanding the mechanism can complicate the picture-for example, the mechanism of cell death is not uniform across all cell types.

"We see some cells where there is an apoptotic response, where we see other outcomes in other cell types. We see activity that's broad, but a variety of different downstream mechanisms appear to be in play. And I think this is not just true for the BET inhibitors; it seems to apply to other chromatin-based targets as well."

Although that provides an opportunity to understand what is going on mechanistically, he said, "it makes it challenging because you're not looking for a singular mechanism; you're looking for the mechanism in a particular context."

Tummino added that understanding the mechanism of some targets may involve looking at effects outside of chromatin regulation. "There are going to be nonhistone substrates for a lot of these enzymes. And some have already been identified. A lot of that work could be quite interesting, and it might be that not all the effects we're seeing in targeting these enzymes or readers are coming at the level of the chromatin. It may be in the cytosol or in the nucleus outside of chromatin," he said.

\section{Mapping potential}

Panelists agreed that expanding chromatin-targeted drug development beyond genomically altered cancers will require increased commitment to chromatin profiling and follow-up functional studies to test new hypotheses.

Chromatin profiling involves mapping the locations and levels of histone modifications throughout the genome.

"The technology we have with ChIP-seq today, and even newer versions of this, are enabling a kind of analysis that's never been seen before," said Schreiber.

Many labs have taken advantage of the fact that genome-sequencing technologies are more widely available and have seen significant reductions in cost in the last decade. 
For example, Bradley Bernstein, who is heading up the global chromatin mapping effort at the Broad Institute, has published protocols for performing nano-ChIP-seq on samples with small numbers of cells. ${ }^{15}$ Bernstein is a senior associate member of the Broad Institute and co-directs its epigenomics program. He also is a professor in the Department of Pathology at Massachusetts General Hospital and Harvard Medical School and an early career scientist at the Howard Hughes Medical Institute.

But Schreiber said that the technology still has a lot of room for improvement. "We need a big leap in the technology to lower the cost and improve the throughput to be able to do single-cell analysis. Right now, it's slow and costly, and so you have to pick and choose," he said.

Cliff Meyer, a research scientist at Dana-Farber, asked the panelists their thoughts on the quality of data in genomewide chromatin profiling studies.

Smith said that it has been a mixed bag. "We've got experience in both directions, where we've had good success merging our datasets with publicly available datasets and getting consistent answers. We've also seen real inconsistencies where we struggle to interpret our data in the context of the publicly available data," he said, which is "likely due to differences in methodologies and experimental conditions."

"My sense is that we probably have a little more confidence in public data than we did two or three years ago," added Tummino. "I remember the discussion then around the nonselectivity of antibodies that were being used for ChIP. I feel like some of the most basic issues like that are being dealt with. We're not there yet, but one could take a little bit of optimism from the progress in the last two or three years that we're getting there."

One effort to improve antibody quality was a 2012 partnership between the SGC and Life Technologies Corp. to make available a master set of recombinant antibodies against epigenetic targets. Thermo Fisher Scientific Inc. acquired Life Technologies earlier this year.

Tummino said that he was impressed by a recent collaboration between the Broad Institute and the Novartis Institutes for BioMedical Research, which used mass spectrometry to map histone methylations in the Cancer Cell Line Encyclopedia. ${ }^{16}$ The method led the team to discover activating mutations in nuclear SET domain-containing protein 2 (MMSET; WHSC1; NSD2).

"This is clearly an example of a highquality, large-scale endeavor that impacts the cancer epigenetics field," said Smith. "And it is also a good example of a productive collaboration between a large pharma and an academic entity."

Roberts was less certain about the discovery potential of the mass spectrometry approach. "I'm torn on how useful this method is going to be. On one hand, we're picking up new things. On the other hand, if it's just picking up a proxy-that is, another marker-driven by a DNA mutation, you could argue we should just sequence more," he said. "But I think it could have the potential to identify real changes in chromatin state that may have a shot at being therapeutically modulated."

\section{Expanding the scope}

Although chromatin-targeted compounds are starting to produce results in cancer, applications in nononcology indications are still far behind. For existing targets such as HDACs and BET bromodomains, developing selective compounds with appropriate safety profiles for chronic use remains a prime challenge.

Mahadevia told SciBX that most people in the field are focused on oncology. "There are fewer folks working outside of oncology, so the pace of identification and validation of new targets is slower. In this 'second act' of epigenetics, you really can't own a space such as CNS or inflammation. You really have to look on a target-by-target basis."

He added, "HDACs are a good example of a target where multiple labs have identified corroborating findings, looking at the impact on things like cognition and the regulation of genes in the hippocampus. There are targets like HATs that are emerging and are getting to that similar level of validation. It's happening in pockets, with a small cadre of investigators in the neurosciences, but it's not happening at the scale that it should."

Other examples include work published by GSK's EpiNova DPU on the role of BET bromodomain proteins in inflammation, and a recent immunology study from Constellation showing that the protein family also regulates $\mathrm{T}$ helper type 17 (Th17) cell function.

Tummino said that there are other scattered cases of intriguing results outside of oncology, including the use of compounds to treat latent viral infections, and work from Harvard pediatric oncology professor Stuart Orkin on reactivating fetal hemoglobin expression to treat sickle-cell anemia.

The challenge will be showing that these targets can be inhibited with a benign safety profile. Donald McCaffrey said that for BET bromodomain inhibitors, improved safety may be possible by increasing selectivity of compounds for specific bromodomains.

McCaffrey is cofounder, president and CEO of Resverlogix Corp. and spinout company Zenith Epigenetics, which is developing BET inhibitors for oncology and autoimmune indications.

Mahadevia said that the same was true for
"We need a big leap in the technology to lower the cost and improve the throughput to be able to do single-cell analysis."

- Stuart Schreiber,

Broad Institute of MIT and Harvard the use of HDACs in particular in chronic neurological diseases. "I think we have to have a very early and intensive focus on improving safety," he said. "Especially in the case of HDACs, selectivity matters both in probing the biology and in ultimately developing a drug to use in populationssuch as patients with mild to moderate cognitive impairment-where people have some of their activity of daily living intact under acetylcholinesterase inhibitor therapy."

Karus' Kerry added, “Tool compounds like Tubastatin have been helpful because they are quite HDAC6 selective. It gives you a fairly 
reasonable idea of what the effects of an inhibitor might be. But designing a selective drug-like compound is challenging as there are no crystal structures of HDAC6."

He also added that it may not always be better to be more isoform selective because you may need activity against other HDACs. He said that it is important to test compounds with a range of potencies and selectivities in preclinical models.

Schreiber said that he would turn to human genetic studies to guide target selection. "I would first turn to germline heritable genetics. I would look at genetic variations associated with any non-oncology disease and see ones that are enriched in chromatin regulators." In those diseases, he added, "I would do deep sequencing to look, for example, for rare protective variants in chromatin regulators associated with some chronic heritable disorder."

Roberts agreed but said that the need for more chromatin profiling is particularly pronounced in diseases outside of oncology. Even though mutations may still be found in genomewide association studies, they cannot be counted on to play the same driving role they have in cancer.

"I think the developmental biology of chromatin state is going to be absolutely key because we're back into correlative biology," he said. "With DNA mutations, we have an absolute result-mutation or no mutation. With chromatin biology, what do you compare it to? We know what our disease state looks like, but we have an extremely poor ability to identify what normal is, and therefore we can't really identify what the aberrancy is."

\section{Chromatin clinic}

The panel and audience members returned frequently to a discussion of clinical development challenges for compounds targeting chromatin regulators.

Richard Gregory, head of Sanofi's Genzyme R\&D center, asked if any tumors have developed resistance to new compounds such as the BET bromodomain inhibitors.

Tummino expects resistance to emerge but said that it is too early to predict resistance mutations. "Our expectation is that there will be resistance that emerges from targeting epigenetic regulators. I think that is a relatively safe assumption. However, we don't have enough clinical experience to have observations of resistance at this point. We are able to generate resistant clones for some of these targets in vitro."

Henry Long, associate director for the Center for Functional Cancer Epigenetics at Dana-Farber, asked whether new compounds might be combined with and synergize with existing drugs.

Schreiber highlighted work from Jeff Settleman on combining HDAC inhibitors with Tarceva erlotinib, an epidermal growth factor receptor 1 (EGFR1; HER1; ErbB1) inhibitor, to treat lung cancer by eliminating persister cells that may cause Tarceva resistance.

"Personally, I think that there's going to be a lot of new and different ideas about combinations," said Schreiber.

Settleman is senior director of discovery oncology at the Genentech Inc. unit of Roche. Astellas Pharma Inc., Chugai Pharmaceutical Co. Ltd. and Roche market Tarceva for pancreatic cancer and non-small cell lung cancer (NSCLC).

Indeed, Audia said that it will be critical to see how new chromatinmodulating therapies work in conjunction with standard of care. "One of the things that we do is to look at the compatibility of our drug with the existing standard of care. We look to see if there's cumulative efficacy or synergistic toxicities to find out if it can be added to standard of care," he said. "We've certainly seen that with EZH2 inhibitors combined with CHOP [cyclophosphamide, hydroxydaunorubicin, oncovin and prednisone]-a very nice tumor regression without any evident preclinical exacerbation of the toxicities."

$\mathrm{He}$ added that rationally designing combination strategies will require better understanding of the signaling pathways downstream of these new compounds.

Panelists agreed that an important factor for advancing new compounds into the clinic will be the ability to collaborate with clinicians. This is not a problem specific to chromatin regulation but is more pronounced because there are so few genetically altered cancers in which compounds are being tested.

Tummino said that if they have an agent with new activity, they would work with clinical scientists to get primary samples and get researchers' input on other models because most preclinical data does not translate well to the clinic.

"We are not just thinking about tumor types but [also] subtypes to understand the relevance of the findings. That is essential; we shouldn't be progressing without that insight."

Roberts agreed. "A lot of the indications we are seeing sensitivity in aren't going to be in a standard panel of cell lines. It really does require expertise from those who do translational work and have expertise in those particular cancer types," he said.

\section{The next frontier}

Finally, panelists discussed the prospects for developing compounds that act by causing cells to differentiate into other cell types rather than by killing cells.

"There are far fewer cell differentiation-type therapies than there are cytotoxic or proapoptotic therapies," Audia said. "ATRA [all-trans retinoic acid] is certainly one of the better examples of a differentiation therapy," he added. But he said that it would be complicated to unravel the biology — in particular in oncology or other indications - in which cell death is commonly the desired outcome.

"I think it will be a challenge to interpret data going forward, if things like histone methyltransferase and histone demethylase inhibitors have more of a differentiation effect. How do we deal with that data, in hematological malignancies in particular, where some of the criteria for response are really based on classic human therapies and wiping out bone marrow?"

"People want to see tumors melt," said Tummino. "Everybody would like that because we've been testing cell-cycle inhibitors and kinase inhibitors, and they're looking for that profile. So part of it is changing that mindset."

He added, "From a mechanistic standpoint, I don't think we have all the tools we need to understand the therapeutic potential of differentiation therapy. We'd like to go in that direction. But then the second part of it is to have a bit of a cultural change within drug discovery. And it's been reasonably positive so far, but there's a lot to do."

"I couldn't agree more," said Schreiber. "I love this, and it's harder because we know less, and it has less precedence. But we have to tackle this."

Cain, C. SciBX 7(19); doi:10.1038/scibx.2014.545

Published online May 15, 2014 


\section{REFERENCES}

1. Lou, K.-J. SciBX 6(40); doi:10.1038/scibx.2013.1118

2. Arrowsmith, C.H. et al. Nat. Rev. Drug Discov. 11, 384-400 (2012)

3. Taunton, J. et al Science 272, 408-411 (1996)

4. Cain, C. SciBX 7(9); doi:10.1038/scibx.2014.245

5. Filippakopoulos, P. et al. Nature 468, 1067-1073 (2010)

6. Nicodeme, E. et al. Nature 468, 1119-1123 (2010)

7. Whyte, W.A. et al. Cell 153, 307-319 (2013)

8. Lovén, J. et al. Cell 153, 320-334 (2013)

9. Cain, C. BioCentury 21(15), A12; April 15, 2013

10. Anders, L. et al. Nat. Biotechnol. 32, 92-96 (2014)

11. Wilson, B.G. et al. Cancer Cell 18, 316-328 (2010)

12. Wang, X. et al. Cancer Res. 69, 8094-8101 (2009)

13. Knutson, S.K. et al. Proc. Natl. Acad. Sci. USA 110, 7922-7927 (2013)

14. Harris, W.J. et. al. Cancer Cell 21, 473-487 (2012)

15. Adli, M. \& Bernstein, B.E. Nat. Protoc. 6, 1656-1668 (2011)

16. Jaffe, J.D. et al. Nat. Genet. 45, 1386-1391 (2013)

COMPANIES AND INSTITUTIONS MENTIONED

Acetylon Pharmaceuticals Inc., Boston, Mass.

Amgen Inc. (NASDAQ:AMGN), Thousand Oaks, Calif.

Astellas Pharma Inc. (Tokyo:4503), Tokyo, Japan

AstraZeneca plc (LSE:AZN; NYSE:AZN), London, U.K.

Atlas Venture, Cambridge, Mass.

Biogen Idec Inc. (NASDAQ:BIIB), Weston, Mass.

Broad Institute of MIT and Harvard, Cambridge, Mass.

Cancer Research UK Manchester Institute, Manchester, U.K.

Chugai Pharmaceutical Co. Ltd. (Tokyo:4519), Tokyo, Japan
Constellation Pharmaceuticals Inc., Cambridge, Mass.

Dana-Farber Cancer Institute, Boston, Mass.

Epizyme Inc. (NASDAQ:EPZM), Cambridge, Mass.

Forma Therapeutics Holdings LLC, Watertown, Mass.

Genentech Inc., South San Francisco, Calif.

GlaxoSmithKline plc (LSE:GSK; NYSE:GSK), London, U.K.

Harvard Medical School, Boston, Mass.

Harvard University, Cambridge, Mass.

Howard Hughes Medical Institute, Chevy Chase, Md.

Johnson \& Johnson (NYSE:JNJ), New Brunswick, N.J.

Karus Therapeutics Ltd., Chilworth, U.K.

Massachusetts General Hospital, Boston, Mass.

Massachusetts Institute of Technology, Cambridge, Mass.

Merck \& Co. Inc. (NYSE:MRK), Whitehouse Station, N.J.

Novartis AG (NYSE:NVS; SIX:NOVN), Basel, Switzerland

Novartis Institutes for BioMedical Research, Cambridge, Mass.

Oryzon Genomics S.A., Barcelona, Spain

RaNa Therapeutics Inc., Cambridge, Mass.

Resverlogix Corp. (TSX:RVX), Calgary, Alberta, Canada

Roche (SIX:ROG; OTCQX:RHHBY), Basel, Switzerland

Rodin Therapeutics Inc., Cambridge, Mass.

Sanofi (Euronext:SAN; NYSE:SNY), Paris, France

Structural Genomics Consortium, Oxford, U.K.

Syros Pharmaceuticals Inc., Watertown, Mass.

Tensha Therapeutics Inc., Cambridge, Mass.

Thermo Fisher Scientific Inc. (NYSE:TMO), Waltham, Mass.

Whitehead Institute for Biomedical Research, Cambridge, Mass.

Zenith Epigenetics Corp., Calgary, Alberta, Canada 